\title{
Multi-Scale Entropy and Neural Networks for Detection of Depth of Anaesthesia within EEG Signals
}

\begin{abstract}
Mohamed H. Almeer
Computer Science \& Engineering Dept, College of Engineering, Qatar University, POB 2713, Qatar

Abstract: In recent years, multi-scale sample entropy (MSSE) is rapidly gaining popularity as an interesting tool for exploring neurophysiological mechanisms. In this paper, we propose a new method based on MSSE for on-line monitoring of the depth of anaesthesia (DoA) to quantify the anaesthetic effect with real-time electroencephalography by using MSSE. Empirical mode decomposition (EMD) was used successfully to filter EEG recordings of artefacts before the imitation of the recognition phases. Artificial neural networks were used to classify three scales of the MSSE into three stages of hypnosis. Results showed $95 \%$ accuracy of the tested clinical anaesthesia EEG recordings obtained for 20 patients.
\end{abstract}

Keywords: DoA; Anaesthesia; EMD; ANN; Sample Entropy.

\section{INTRODUCTION}

Electroencephalogram (EEG) signals are a proven method for monitoring the depth of anaesthesia (DoA). In general, many anaesthesiologists monitor the depth of anaesthesia by means of clinical parameters such as blood pressure, heart rate, muscle movement, pupil size, lacrimation, and airway pressure[1-3]. However, these physiological parameters change in response to anaesthetic agents and are also affected by other factors such as body weight and age. Therefore, these parameters alone may not provide an accurate assessment of anaesthesia. Since anaesthetic agents affect the brain cortex, monitoring brain activity using EEG signals would be a suitable method to measure the DoA. EEG signals are generally non-stationary, although they may include some stationary epochs; these signals reflect the non-stationary activity of the brain.

Many studies have been conducted on the measurement of DoA using various methods and with multiple algorithms [21, 22, 25]. Neural networks [10,11, 14, 15, 17, 18, 20], both feed-forward and recurrent, as well as DWT wavelets $[8,11]$ have also been used to classify the anaesthesia levels. Empirical mode decomposition (EMD) [11] and its variants have been employed to filter the samples of artefacts and obtain a clean EEG before attempting recognition. Some studies have investigated the value of different frequency bands in EEG [16]in the measurement of the DoA; for example, the alpha-frequency band showed a strong correlation to the level of consciousness during surgery under anaesthesia. Furthermore, some studies have utilised the chaotic feature as described by the chaos theory to follow the hypnotic levels [9].

Many scientific standards have been developed recently to measure DoA, but the most preferred important monitor is the bispectral analysis index (BIS) [4, 5]. BIS, one of the high-grade spectral analysis methods, is a complex measure encompassing the domains of time and frequency as well as high-order spectral parameters. It is determined by the analysis of the correlation of the different phases of the parts of the EEG signal. Generally, BIS readings are obtained from signals of four different EEG electrodes. BIS monitors are the most common tools used to measure the DoA, except in the case of some anaesthetic drugs. However, some investigations have indicated that the patient could awaken momentarily even if the BIS reading is between 40 and 60 .

BIS entropy monitors have been recently developed to monitor the DoA using EEG signals. These monitors mainly display two readings simultaneously: response entropy (RE) and state entropy (SE) 
[27]. Both parameters reflect the degree of regularity (or irregularity) of the EEG signal. The RE and SE measures are based on spectral entropy which relies heavily on fast Fourier transformation (FFT)for the calculations [28]; therefore, the spectral entropy may miss some nonlinear and nonstationary information within the EEG signal.

Approximate entropy (ApEn) [30] and sample entropy (SampEn) [31] are two recently developed and widely used entropy measures that have been shown to be efficient in the detection of complexity of any time series. In particular, SampEn has been shown to perform even better than ApEn in detecting the DoA of patients in surgeries [29-31]. SampEn measures complexity based on only one time scale, which makes it likely to miss some features. On the other hand, multi-scale entropy (MSE) [7, 13, 23, $33,34,35]$ used multiple scales to determine the complexity and can therefore capture the dynamic changes associated with multiple time scales. This property makes MSE much more suitable than other techniques in computing the degree of complexity for the EEG time series.

Although the use of MSE improves the correlation between the hypnosis level and the entropy value, but ultimately its is a value between 0 and 3. Because of these multiple entropy values, this method does not allow for a straightforward assessment of the DoA [35]. Artificial neural network (ANN)is one of the best artificial intelligence methods that has the ability to learn from a set of presented data through matching inputs with the outputs and then generalise the learned relationship to other foreign inputs [36]. By updating unknown weights and functions until a match is reached, a good relation is constructed on the basis of the weights and functions that correlate the input to the output sets.

The purpose of this research study is to estimate the level of anaesthesia based on EEG time series using multi-scale SampEn with the help of an ANN model that maps the three scales of SampEn to the anaesthetic condition of the patient under surgery, by using only generic filtering obtained by the generic EMD algorithm.

\section{Material ANd Methods}

\subsection{Data Source}

The single-channel EEG recording tested in this study was collected from 20 patients aged between 23 and 70 years through a forehead-mounted sensor placed on patients went under surgery of ENT cases under general anaesthesia at the National Taiwan University Hospital (NTUH) of Taiwan. The EEG data recording has been acquired using Philips Intellivue MP60. The anaesthetic drug sevoflurane or sesflurane was used for tracheal intubation of all 20 patients.

\subsection{Data Processing}

The standard operation procedure with general anaesthesia was divided into 4 stages: pre-operation, induction, maintenance, and recovery. The collected EEG recordings were divided to 2 parts. One set of recordings was collected from 6 patients to estimate the distinction between different anaesthesia levels since they show clear separation boundaries, can help determine the sensitivity level of MSE for the generated time indices, and for the neural network training. The other set of recordings was used for the testing and validation of the proposed neural prediction system.

The EEG signals were recorded with a sampling frequency of $128 \mathrm{~Hz}$ (samples per second) and its microvolt readings were digitised using 12-bit analogue-to-digital converter, thus giving a scalar range between 0 and $2^{12}=4096$ values. Figure 1 shows some samples for an EEG recording during general anaesthesia covering three anaesthetic levels in addition to a recovery level, as shown below.

\subsection{Expert Assessment of Consciousness Level}

During the period of anaesthesia, the patients were observed by two research nurses who carefully observed the signs of unconsciousness and recorded the vital parameters such as heart rate and arterial blood pressure besides other clinical signs. Further, they recorded the time points and durations of various anaesthetic events such as induction, muscle signs; stimulus and clinical signs of the patients; and the duration of surgery. 


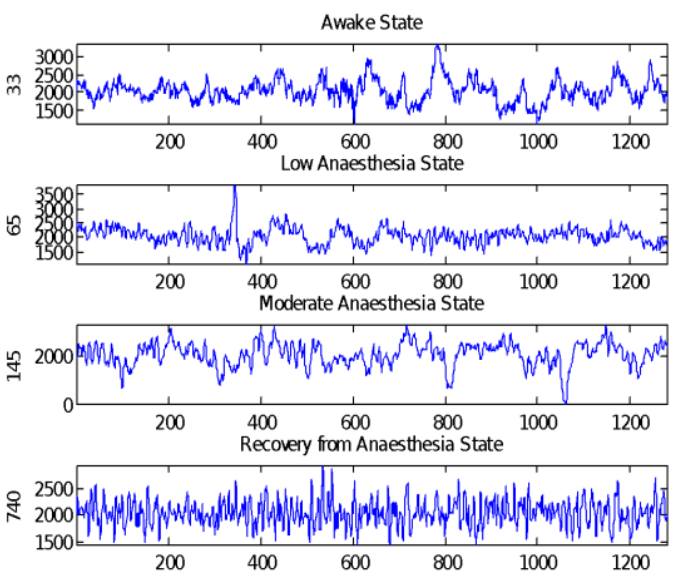

Figure1. Four single-channel EEG epochs, each of 10 seconds, representing brain function at different depths of anaesthesia. $X$-axis represents the epoch index ( /10 seconds) and y-axis represents the captured epochs index (1-840).

Five expert anaesthesiologists were then asked to score the DoA of the patient on a scale of 10 to 100 based on the recordings of the patient's signs only. A non-correlated and independent decision was then collected from each expert solely based on the data provided, without any contact with the patients. Finally, the average of the opinions of the 5 experts was determined by plotting on a graph, with time on the $\mathrm{X}$-axis and their scores (from 10 to 100 for consistency the BIS-based anaesthesia level) on the Y-axis.

The standard for anaesthetic level defined as per the BIS machines is explained in Table 1. Values between 40 and 60 are defined as 'anaesthetic depth suitable for surgery', those below 40 indicate that the anaesthesia is deep, and those between 60 and 85 indicate that the anaesthesia is light and may be suitable only for some types of surgeries.

Table1. Range of BIS value for different levels for depths of anaesthesia

\begin{tabular}{|l|l|l|}
\hline $\begin{array}{l}\text { Depth of Anaesthesia } \\
\text { (Consciousness Level) }\end{array}$ & Level Description & Range \\
\hline Awake & & BIS $(100-80)$ \\
\hline Light Anaesthesia & & BIS 80-60 \\
\hline Moderate Anaesthesia & Suitable for Surgery & BIS $40-60$ \\
\hline Deep Anaesthesia & & BIS $10-40$ \\
\hline
\end{tabular}

Finally, it is noteworthy that in this study, 20 anaesthesia charts were digitised and were used as a gold standard for determining the DoA and were used to train the neural network as training targets.

\subsection{Sample and Multi-Scale Entropy}

SampEn [44] is a modification of ApEn; both are used to examine physiological time series signals such as EEG and other signals. SampEn is independent of the data length and can be implanted more easily in software but is computationally different. In $A p E n_{2}$ the comparison between the template vector and the rest of vectors includes the comparison with itself, and it is always possible to have the logarithm of the probability.

SampEn is a measure of complexity but does not include self-similar patterns as found in ApEn. As in $A p E n$, if an embedding dimension $\mathrm{m}$ is given as well as tolerance $\mathrm{r}$ and number of the data points $\mathrm{N}$, then we can define SampEn as negative logarithm of the probability that two sets of similar data points of length $m$ have for distance $<r$. Then, two sets of similar data points of length $m+1$ also have distance $<$ rare and represented by

$\operatorname{SampEn}(\mathrm{m}, \mathrm{r}, \mathrm{N})$, or by $\operatorname{SampEn}(\mathrm{m}, \mathrm{r}, \mathrm{t}, \mathrm{N})$

Where $\mathrm{t}$ is the sampling time.

If a time-series data set of length $\mathrm{N}=\left\{\mathrm{x}_{1}, \mathrm{x}_{2}, \mathrm{x}_{3}, \ldots, \mathrm{x}_{\mathrm{N}}\right\}$ with a constant time interval $\tau$, we define a template vector of length $\mathrm{m}$, such that $\mathrm{X}_{\mathrm{m}}(\mathrm{i})=\left\{\mathrm{x}_{\mathrm{im}} \mathrm{x}_{\mathrm{i}+1}, \ldots, \mathrm{X}_{\mathrm{i}+\mathrm{m}-1}\right\}$ have Chebyshev or Euclidean 
distance. Then, compute the total distances $\angle \mathrm{R}=\mathrm{r} * \mathrm{SD}$ (standard deviation of original signal). The distance function has the formula:

$\mathrm{d}[\mathrm{Xm}(\mathrm{i}), \mathrm{Xm}(\mathrm{j})]<\mathrm{r}$, and $\mathrm{i} \neq \mathrm{j}$

We compute the number of vector pairs in template vector of length $m$ and $m+1$ having $d[X m(i)$, $\mathrm{Xm}(\mathrm{j})]<\mathrm{r}$. We can define the sample entropy:

SampEn $=-\log (\mathrm{A} / \mathrm{B})$

where

$A=$ number of template vector pairs having $d[X m(i), X m(j)]<r$ of length $m+1$ and

$\mathrm{B}=$ number of template vector pairs having $\mathrm{d}[\mathrm{Xm}(\mathrm{i}), \mathrm{Xm}(\mathrm{j})]<\mathrm{r}$ of length $\mathrm{m}$.

Therefore, $\operatorname{SampEn}(m, r, \tau)$ will be always be either zero or any other positive value. A smaller value of SampEn also indicates more self-similarity in data set or less noise.

Previous studies on SampEn have been shown that $\mathrm{m}=1$ or 2 would be appropriate; further, having $\mathrm{r}$ $=0.1$ to $0.2 \mathrm{SD}$ ( $\mathrm{SD}=$ Standard Deviation) are more suitable values for clinical data analysis.

Traditional entropy measures quantify only the regularity (predictability) of time series based on a single scale. There is no direct correlation between regularity and complexity. Neither completely predictable or periodic signals, which have minimum entropy, nor completely unpredictable or uncorrelated random signals, which have maximum entropy, are actually complex. There is no consensus on the definition of complexity. Intuitively, complexity can be defined as the correlation over time and space scales.

The MSE method incorporates two aspects. First, a coarse graining process is applied to the time series data by averaging data within non-overlapping windows but of increasing length. The coarsegrained element $\mathrm{y}_{\mathrm{j}}^{(\tau)}$

$y_{j}^{(\tau)}=\frac{1}{\tau} \sum_{i=(j-1) \tau+1}^{j \tau} \quad x_{i}$

Where $\tau$ represents the scale factor and $1 \leq \mathrm{j} \leq \mathrm{N} / \tau$. The length of each coarse-grained time series is $\mathrm{N} / \tau$.
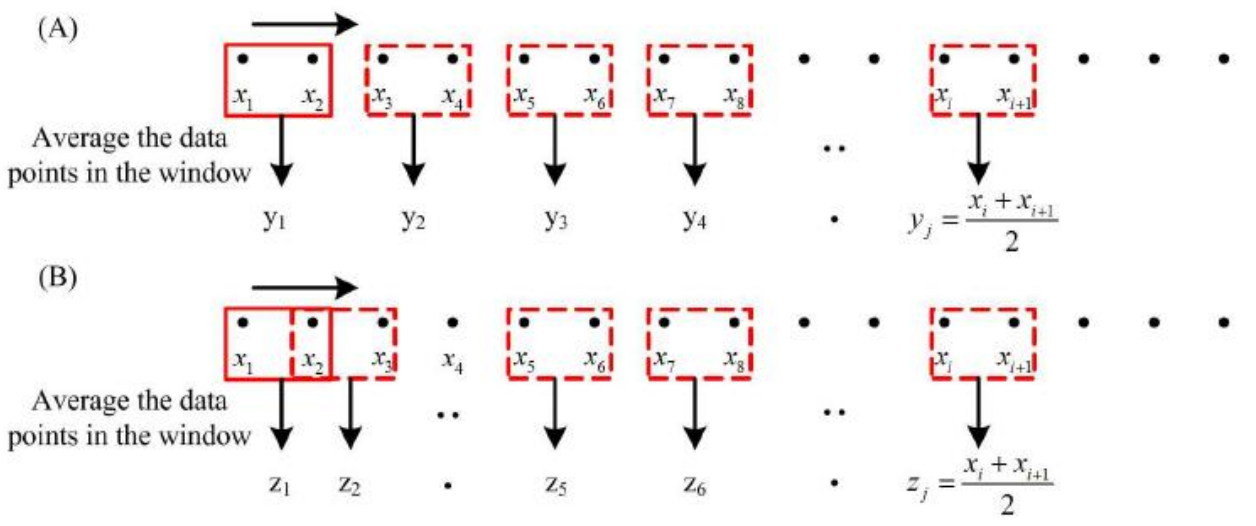

Figure2. An illustrations of multiscale decomposition procedures. (a) Coarse-graining procedure and (b) Moving average procedure [45].

Second, SampEn is calculated for each coarse-grained time series and then plotted as a function of the scale factor. SampEn is a 'regularity statistic'. It 'looks for patterns' in a time series and quantifies its degree of predictability or regularity (see Figure 2).

\subsection{Empirical Mode Decomposition (EMD)}

EMD is an adaptive method to decompose a non-linear or non-stationary time series signal into its intrinsic mode functions (IMF) [46]. This process to obtain the IMFs is called sifting, and it can be outlined into the next phases: 
1. Create upper envelope $E_{u}(t)$ by local maxima and lower envelope $E_{l}(t)$ by local minima of data $x(t)$.

2. Calculate the mean of upper and lower envelope

$m_{1}(t)=\frac{E_{u 1}(t)+E_{l 1}(t)}{2}$

3. Subtract the mean from original data

$h_{l}(t)=x(t)-m_{l}(t)$

4. Verify that $\mathrm{h} 1(\mathrm{t})$ satisfies conditions for IMFs. Repeat steps 1 to 4 with $\mathrm{h} 1(\mathrm{t})$, until it is an IMF.

5. Get first IMF (after k iterations)

$c_{l}(t)=h_{l(k-l)}(t)-m_{l k}(t)$

6. Calculate first residue

$r_{l}(t)=x(t)-c_{l}(t)$

7. Repeat whole algorithm with $r l(t), r 2(t), \ldots$ until residue is monotonic function.

8. After $\mathrm{n}$ iterations, $x(t)$ is decomposed per equation (1).

The decomposition process can be stopped when $r_{n}$ becomes a monotonic function, i.e., no more IMF can be extracted from it. Nevertheless, it is known that only a determined number of IMFs have physical meaning, and it is only important to apply a certain number of IMFs to extract relevant information from the main signal.

\subsection{Artificial Neural Networks (ANNs)}

ANNs are considered to be excellent classifiers due to their properties of adaptive learning, robustness to signal distortion, and good generalisation effect. For the classification of the anaesthetic level, a Feed-Forward Back Propagation Multi-Layer Perceptron was chosen. This particular type of neural networks is considered a 'de facto' and is widely known in neural classification and recognition types of applications. Since it has multiple layers and nonlinear activation functions (tan sigmoid or log sigmoid), the network can learn and recognise nonlinear beside linear correlations between the input and the output sets. The proposed neural network is shown in Figure 3.

The feed-forward network has one or more hidden layers beside the input and output layers. With those multiple layers of neurons, each utilising non-linear activation function enables the network to learn non-linear relation(s) between the input and output sets. Each neuron on different layers has a standard architecture which depends on storing multiple weights, a non-linear activation function, and a single bias. The following equation simplifies the equation core of the neurons:

$Y=f\left(\sum_{j=1}^{N} w_{j} \cdot I_{j}\right)$

where $\mathrm{f}$ is the required activation function that could be one of the next two functions: LogSig activation function or TanSig log activation function.

$\operatorname{LogSig}(\mathrm{n})=1 /(1+\exp (-\mathrm{n}))$
$\operatorname{TanSig}(\mathrm{n})=2 /\left(1+\exp \left(-2^{*} \mathrm{n}\right)\right)-1$

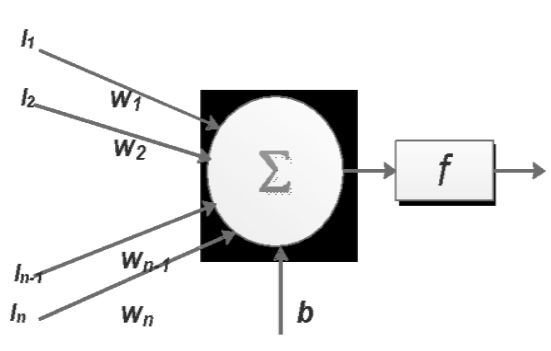

A.

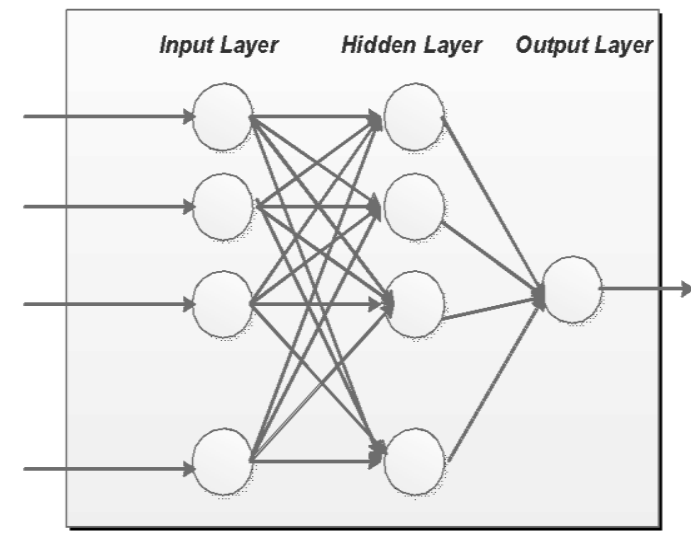

B.

Figure3. Neural network for recognition of depth of anaesthesia. (A) shows one perceptron; while (B) shows a 3-layers multi-perceptron neural network. 


\section{RESUlTS}

In this study, some aspects of the raw EEG recording have prompted us to perform an early sorting of both trained and tested EEG recordings. The sorting was necessary because of the nature of the EEG signals itself. The signals showed 2 unpleasant properties: the artefact contamination from the patient signals themselves, as observed in electrocardiograms and electromyograms, as well as some signal discontinuity experienced during the EEG recording due to the patient's movements as well as refixing of the electrodes and other unknown sources.

The artefacts and non-continuity in the recorded signal distort the original signal to a level that the SampEn gives misleading outputs, while bearing in mind the high sensitivity of such SampEn algorithm toward noise. However, considering the cleaning of the EEG recording, the application of EMD (Empirical Mode Decomposition)or any of its counters (such as EEMD, CEEMD, or MEMD) for filtration would shorten the amplitude of the signal such that it makes it difficult to discriminate between anaesthetic levels copied from SampEn by visual inspection although these EMD algorithms can clean and filter the signal to some extent. Because of this property, it was decided to compare two cases simultaneously: one with generic EMD filtering and one with no filtering.

\subsection{Phase_1: Choosing the Appropriate Sections of the EEG Signals}

All the patients' EEG recording were carefully screened in two stages - first by visual inspection with the help of Matlab plotting method to identify then discard all the distorted, the non-continuous, clipped, and weak (less than threshold) epochs and then a second phase when SampEn of the whole patient is experimentally calculated and the Doctor Plot for consciousness level (1 to 100) is brought. From some careful entropy screening, it was noted that the entropy of the patient will change between 0.1 and 2.2. Next, all the patterns within specific regions where SampEn did not match with the expert's plot were removed, and the remaining blocks which abide with the expert's plot were taken to complete the training set with. Table- 6 lists all the 6 patients' appropriate blocks that were taken in the training process. From the table, it can be seen that the epoch is a 10 -second recording of EEG signal yielding 1280 samples with $128 \mathrm{~Hz}$ sampling frequency.

Table2. Range of BIS value taken for different levels of depth of anaesthesia

\begin{tabular}{|c|c|c|}
\hline $\begin{array}{c}\text { Depth of Anaesthesia } \\
\text { (Consciousness Level) }\end{array}$ & Level Description & Range \\
\hline A wake (C1) & & BIS (85 - 100) \\
\hline Light Anaesthesia (C2) & & BIS (60- 85) \\
\hline Moderate Anaesthesia (C3) & Suitable for Surgery & BIS (40 - 60) \\
\hline
\end{tabular}

The common anaesthetic level which classifies the patient depth of consciousness is detailed in Table 1.However, since all our patient's records showed that $D o A$ is between 45 and 100, we have reduced the levels to only three, as listed in Table-2.

\subsection{Phase_2: Statistical Analysis of the Trained Set:}

Statistical analysis was carried out on the selected training patterns. First, we extracted the standard deviation, mean, covariance, and the maximum with the minimum coverage of all the samples in each anaesthesia level.

The MSSE measurements were calculated on 6 patients' EEG records at 5 scales for the DoA. Measurements were computed over a window of 10 seconds with no overlap with and without EMD filtering. Figure 4 shows the MSSE for 4 patients with and without EMD filtering.

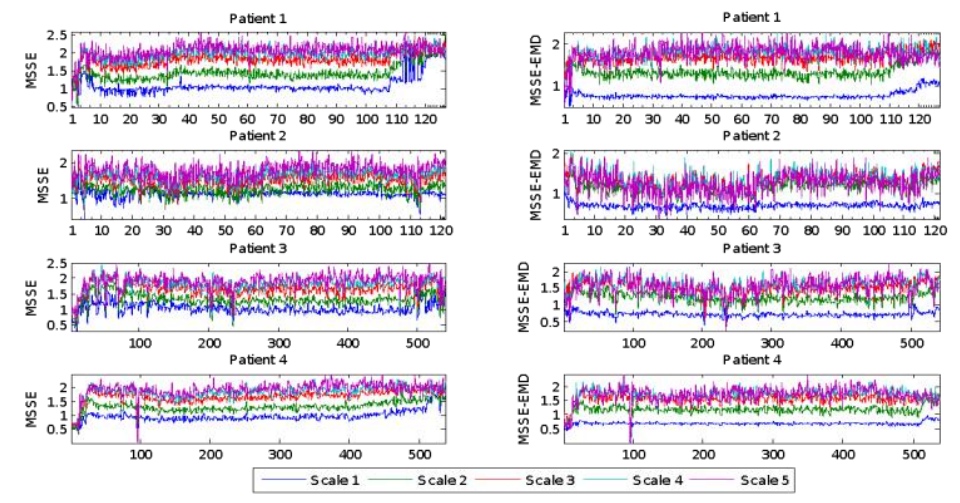

Figure4. Multi-scale sample entropy for four patients with and without EMD 
To clearly investigate the trends in signal variation, median values of all the indices from the 6 patients in two states (Awake and Unconscious) were plotted together for comparison (Figure 5)and visualised by boxplot plotting. Figure 5 has 4 subplots; the first row has 2 plots of Awake and 'Unconscious' when no EMD filtering was applied. It is obviously seen that the mean for the 'Conscious' state was around 1.0 and almost exhibits no change when scales 2 to 5 are observed. The variation range (minimum to maximum) becomes relatively larger from scale1 to 5 .

The bottom part of the Figure shows a similar boxplot for the sample entropy for 5 scales with EMD filtering. It is seen that the trend of the 5 means are clearly different for the 'Awake' state: starts from SampEn $=0.8$ for scale 1 and increases until scale 3; then, from scale 4 to scale 5 , it decreases to around $\operatorname{SampEn}=1.5$. The 'Unconscious' mean level trend has a logarithmic shape; it starts from 0.70 for scale number1;raises until it settles near a value of 1.5 with scale numbers from 2 to 5 ; and then it settles.

It is evident from the previous boxplot that the boundaries of the SampEn for the 3 classes are distinguishable for the time scale 1 as well as indices 2 and 3. Table 3 outlines the means, standard deviations, covariance, and max-min values of the 3 state boundaries. It is also noticeable that the variation of SampEn increases as the scale number increases.

Table3. Different statistical properties outlining the 3 different classes of anaesthesia

\begin{tabular}{|l|c|c|c|}
\hline \multicolumn{1}{|c|}{ Statistical Measurement Name } & Class 1 & Class 2 & Class 3 \\
\hline \multirow{3}{*}{ Mean } & $\mathbf{1 . 5 1 1 7}$ & $\mathbf{1 . 2 5 4 5}$ & $\mathbf{0 . 9 8 8 7}$ \\
& 1.4395 & 1.6493 & 1.2573 \\
\hline \multirow{3}{*}{ Standard Deviation } & 1.6575 & 1.9802 & 1.5832 \\
\hline \multirow{2}{*}{ Covariance } & $\mathbf{0 . 0 8 6 4}$ & $\mathbf{0 . 0 4 7 8}$ & $\mathbf{0 . 1 1 8 8}$ \\
& 0.2209 & 0.1855 & 0.1120 \\
\hline \multirow{2}{*}{ Lower to Upper Bound } & 0.2980 & 0.1545 & 0.1420 \\
& $\mathbf{0 . 0 0 7 5}$ & $\mathbf{0 . 0 0 2 3}$ & $\mathbf{0 . 0 1 4 1}$ \\
& 0.0488 & 0.0344 & 0.0125 \\
\hline
\end{tabular}

This verified that entropy (or scale $1 \mathrm{MSSE}$ ) can make a low distinction between the 'Awake', 'Light Anaesthesia', and 'Unconscious' states, although the best distinction is noticeable between the 'Awake' and 'Unconscious' states. Nevertheless, MSSE has much better distinction ability between these two states when all five scales are observed. The neural network classifier (mentioned in the next few section) will play the main role in recognising different states of anaesthesia based on different scales of the MSSE.

\subsection{Phase_3: Organising and Sorting the Extracted Epochs for Training}

To accurately extract the best representation for $D o A$ from EEG recordings, careful selection of the appropriate epochs should be undertaken. Sequences of EEG Blocks should be chosen for artefactclean samples and those with good SampEn readings that strongly correlate to the expert's decision about the DoA.

As shown from Table 1, the data of the 6 selected patients (Table 6) had recordings that clearly cover the 100 to 40 BIS level readings with sufficient number of blocks covering the 3 chosen consciousness states, in order to ensure the general diversity needed for successful training. Besides, the chosen blocks for the same class showed some acceptable correlation in SampEn values.

From the six patients' EEG recordings, we obtained only 169 blocks of EEG recording, each of 10 seconds, for the first class (BIS 100 to 85);224 blocks for the second class (BIS 85 to 62) and 770 blocks representing the third class (BIS 40 to 60 ). Then, all those selected epochs were inserted in 2-D arrays (3-time indexed scaled entropy readings for each block giving an array depth of 1163 lines by 3 columns), which is called the main training database (MTDB). A corresponding output vector has been prepared such that it gives a reading of 0.9 corresponding to input class one, a reading of 0.5 for a class two, and a reading of 0.25 for class three. This is the vector target that the training should reach within a small error boundary. That output vector will be added to the reconstructed MTDB table (Table 6). 

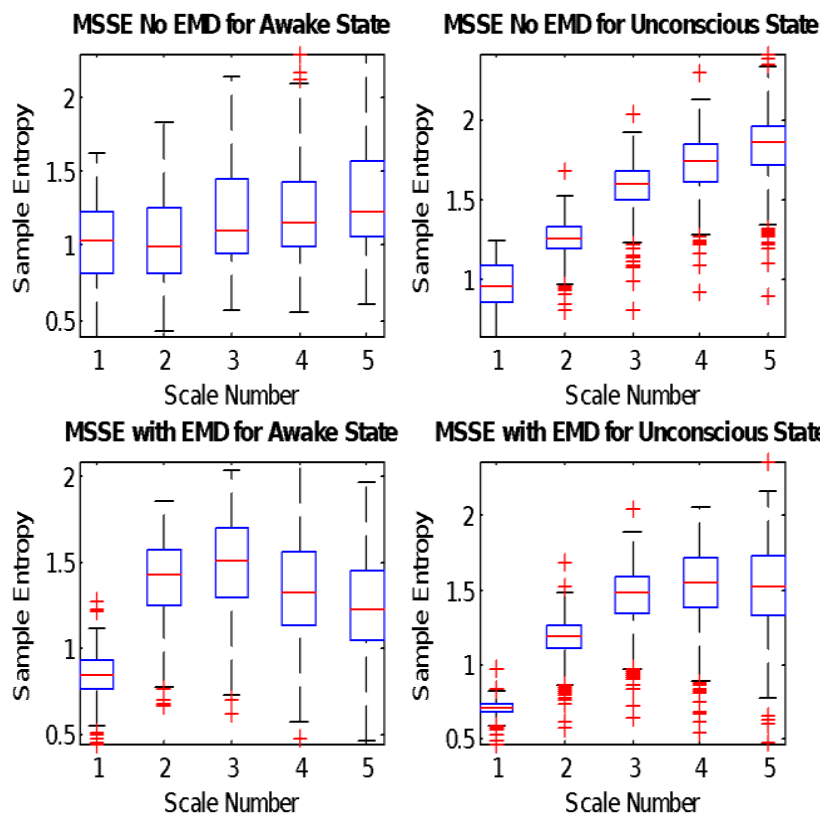

Figure5. The statistical box plots for MSSE with EMD and without EMD, at scales 1, 2, 3, 4, and 5 in the awake state (upper row) and unconsciousness state (lower row).

\subsection{Phase_4: Training the network}

From the MSE values, only 3 time indices levels were considered as inputs for the ANN(NN Tools the neural network toolbox in Matlab). The output target was the depth of anaesthesia score assigned by the experts in the plot after digitisation. Data from six patients out of 20 were collected to train the ANN with, and data from the remaining 14 patients were used to test the ANN.

A multi-layer perceptron network was chosen for its good generalisation effect, with 30 hidden neurons in its first layer employing TAN activation function and another 50 neurons in the second layer with TAN activation function. A single neuron in the output layer with LOG activation function was dedicated to give the output ranges between 0.1 to 0.9 .

The network was trained using back propagation method with a TRAINRP training function and LEARNGDM for its adaptation learning function. The performance function was MSE (Mean Square Error). The epochs reached and the training goal for the three networks is listed in Table 4.

Table4. Feed-Forward Back-Propagation Neural Network training parameters

\begin{tabular}{|c|c|c|c|}
\hline Description & Neural Network 1 & Neural Network 2 & Neural Network 3 \\
\hline Learning Criteria & $\begin{array}{c}\text { 3 Levels, } \\
\text { MSSampEn, } \\
\text { EMD }\end{array}$ & $\begin{array}{c}\text { 2 levels, } \\
\text { MSSampEn, } \\
\text { No EMD }\end{array}$ & $\begin{array}{c}\text { MSSampels, } \\
\text { EMD },\end{array}$ \\
\hline Anaesthetic Levels & 3 & 2 & 2 \\
\hline Number of Layers & $1+2+1$ & $1+2+1$ & $1+2+1$ \\
\hline Neurons in Input Layer & 3 & 3 & 3 \\
\hline Neurons in Hidden Layer 1 and 2 & $30,50($ Tan $)$ & $30,50($ Tan $)$ & $30,50($ Tan $)$ \\
\hline Neurons in Output Layers & $1($ Log $)$ & $1($ Log $)$ & $1($ Log $)$ \\
\hline Training Epochs & 75,776 & 108 & 100,000 \\
\hline Training Goal Reached & 0.00406 & 0.00048 & 0.0021 \\
\hline
\end{tabular}

\subsection{Phase_5: Testing the network on the remaining patients' records}

The neural network was trained with the MSE entropy values of 3 time scales obtained from preprocessed EEG segments (epochs of 1280 sample of $10 \mathrm{~s}$ duration). A total number of 2639 prepared testing patterns of 10-second duration corresponding to the remaining 15 patients were used as a test data set for evaluating the performance of the neural network.

The classification results are shown in Table-5 for three networks: NW1 is a model for recognising 3 levels of anaesthesia when no EMD filtering is enabled; NW2 is a model for recognising 2 levels of 
anaesthesia without embedding EMD filtering; and NW3 is the same as NW2 but EMD filtering is implemented. Figure 6 shows the output of the neural network model corresponding to the 2 and 3 levels of anaesthesia when tested with the unseen test data of 2639 test patterns. Figure 7 shows the first 400 patterns of each output.

In addition, Table 5 shows the accuracy of the recognition for each anaesthetic level. The performance of the ANN is evaluated using two parameters: sensitivity for each anaesthetic level and overall accuracy. The following equations show the performance evaluation parameters formulae.

Sensitivity $_{\text {Awake }}=\frac{\text { Correctly detected Awake anaest hesia }}{\text { Total of actual Awake anaest hesia }} .100$
Sensitivity $_{\text {Low Anaest hesia }}=\frac{\text { Correctly detected Low anaest hesia }}{\text { Total of actual Low anaest hesia }} \cdot 100$
Sensitivity ${ }_{\text {Moderate Anaest hesia }}=\frac{\text { Correctly detected Moderate anaest hesia }}{\text { Total of actual Moder ate anaest hesia }} .100$

Overall Accuracy Total $=\frac{\text { Total Correctly detected anaest hesia levels }}{\text { Total of applied anaest hesia levels }} .100$

Table5. Results of classification of depth of anaesthesia

\begin{tabular}{|c|c|c|c|c|}
\hline $\begin{array}{c}\text { Anaesthetic Level } \\
\text { Class }\end{array}$ & $\begin{array}{c}\text { Total Applied } \\
\text { Patterns }\end{array}$ & \multicolumn{3}{|c|}{$\begin{array}{c}\text { Correctly Detected Patterns } \\
\text { with Class Sensitivity }\end{array}$} \\
\hline $\begin{array}{c}\text { Wake } \\
\text { BIS100-85 }\end{array}$ & 98 & $85(86 \%)$ & $73(74 \%)$ & NW1 \\
\hline $\begin{array}{c}\text { Light Anaesthesia } \\
\text { BIS 85-62 }\end{array}$ & 68 & $18(26 \%)$ & --- & -- \\
\hline $\begin{array}{c}\text { Surgery } \\
\text { Anaesthesia } \\
\text { BIS 60-40 }\end{array}$ & 2473 & 1801 & 2250 & 2347 \\
\hline $\begin{array}{c}\text { Over all Accuracy } \\
\text { with Sensitivity }\end{array}$ & $\mathbf{2 6 3 9}$ & $(72 \%)$ & $(91 \%)$ & $(95 \%)$ \\
\hline
\end{tabular}

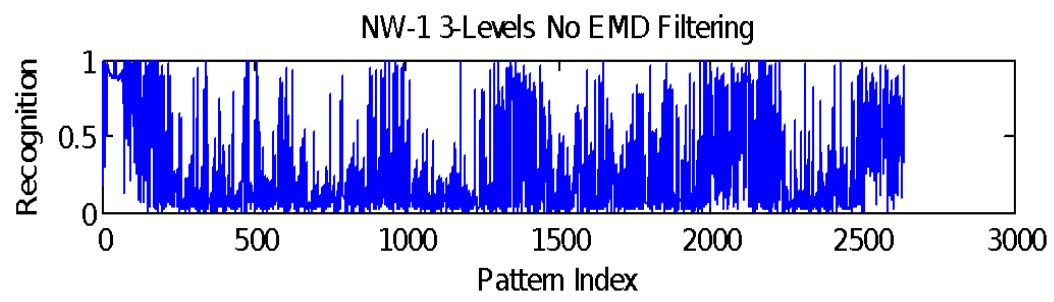

NW-2 2-Levels No EMD Filtering

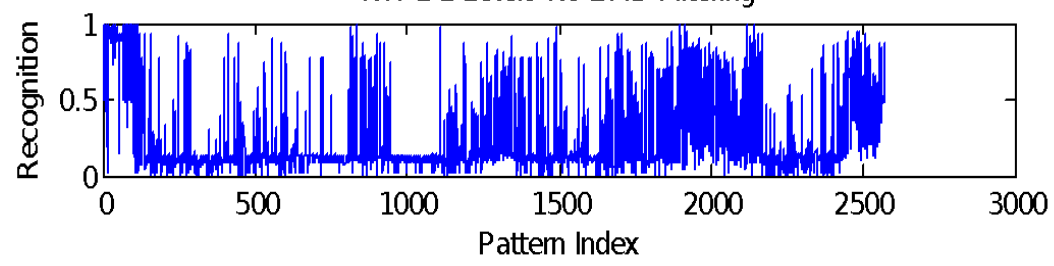

NW-3 2-Levels With EMD Filtering

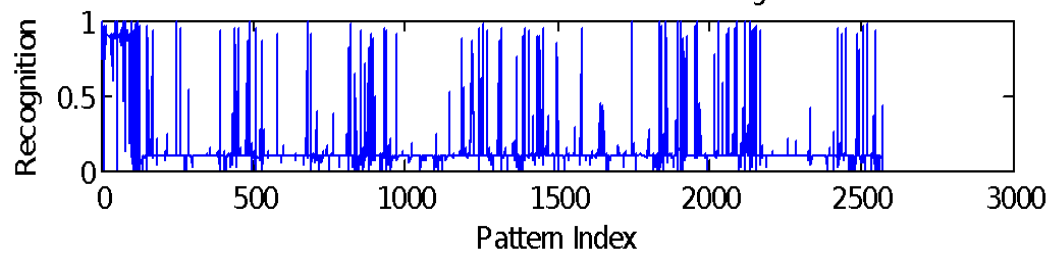

Figure6. Neural network output for the three networks: 3 levels without filtering and 2 levels without and with filtering. 

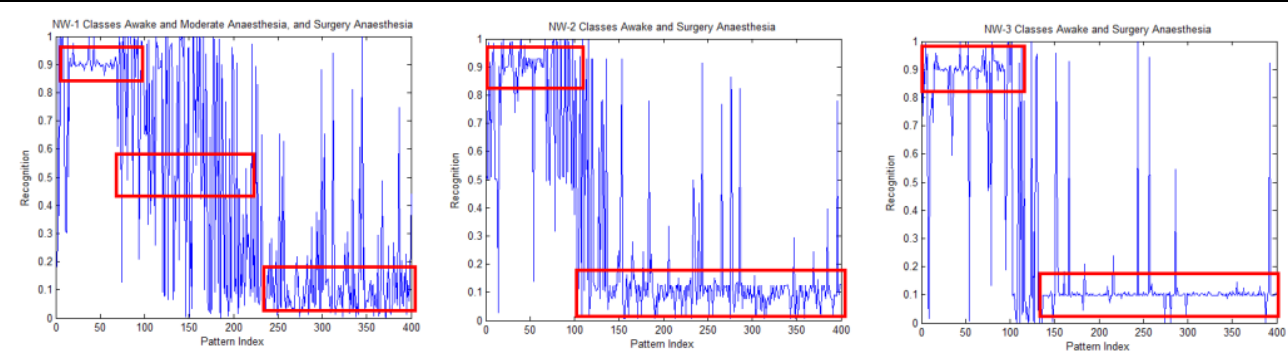

Figure7. The 3 network outputs for the 400 test patterns: patterns [1-98] for class 'Awake', and patterns [99400] for class 'Moderate Anesthesia', while those for the first subplot 3 classes are represented; and finally the previous two beside 'Light Anaesthesia' [99-167]. The red rectangles shows the three classes for each network.

Table6. Records for the six selected patients that were used for training, with their IDs, total epochs, surgery time, and the chosen epochs indices. Numbers (1, 2, and 3) in small font indicate the DoA three levels.

\begin{tabular}{|c|c|c|c|c|c|}
\hline Event & $\begin{array}{c}\text { Patient EEG Record } \\
\text { ID }\end{array}$ & $\begin{array}{c}\text { Total } \\
\text { number of } \\
\text { Epochs }\end{array}$ & $\begin{array}{l}\text { Surgery } \\
\text { Time }\end{array}$ & $\begin{array}{l}\text { Actual Epochs } \\
\text { Range* }\end{array}$ & $\begin{array}{c}\text { Appropriate Epochs Extracted } \\
\text { (1280 Samples each) }\end{array}$ \\
\hline Patient 1 & 1017-61-1106 & 918 & $\begin{array}{l}11: 07- \\
13: 40\end{array}$ & $\begin{array}{c}{[1 \text { to } 60]^{1}} \\
{[61 \text { to } 75]^{2}} \\
{[780 \text { to } 840]^{2}} \\
{[76 \text { to } 285]^{3}} \\
{[286 \text { to } 675]^{3}}\end{array}$ & $\begin{array}{c}{[23,33,41,55,58,56,57,60,} \\
61]^{1} \\
{[65,69,81]^{2},[728 \text { to } 751]^{2}} \\
{[767 \text { to } 772]^{2},[802 \text { to } 839]^{2}} \\
{[90 \text { to } 95]^{3},[120 \text { to } 123]^{3}} \\
{[109 \text { to } 112]^{3},[128 \text { to } 141]^{3}} \\
{[145 \text { to } 188]^{3},[192 \text { to } 243]^{3}} \\
{[274 \text { to } 303]^{3},[308 \text { to } 361]^{3}} \\
{[370 \text { to } 412]^{3},[370 \text { to } 412]^{3}} \\
{[431 \text { to } 478]^{3},[486 \text { to } 515]^{3}} \\
{[548 \text { to } 560]^{3},[579 \text { to } 597]^{3}} \\
{[625 \text { to } 637]^{3}}\end{array}$ \\
\hline Patient 2 & $1017-61-1348$ & 954 & $\begin{array}{l}13: 51- \\
16: 30\end{array}$ & $\begin{array}{l}{[1 \text { to } 30]^{1}} \\
{[35 \text { to } 45]^{2}} \\
{[48 \text { to } 894]^{3}}\end{array}$ & $\begin{array}{c}{[14,17,21,23,31]^{1}} \\
{[278 \text { to } 302]^{3},[308 \text { to } 346]^{3}} \\
{[452 \text { to } 481]^{3},[500 \text { to } 541]^{3}} \\
{[552 \text { to } 601]^{3},[611 \text { to } 659]^{3}} \\
{[704 \text { to } 721]^{3},[728 \text { to } 753]^{3}}\end{array}$ \\
\hline Patient 3 & $1104-23-1345$ & 816 & $\begin{array}{l}13: 46- \\
15: 30\end{array}$ & $\begin{array}{c}{[1 \text { to } 38]^{1}} \\
{[42 \text { to } 63]^{2}} \\
{[588 \text { to } 630]^{2}} \\
{[72 \text { to } 573]^{3}}\end{array}$ & $\begin{array}{c}{[48,50,62,63,67]^{2}} \\
{[576,578,582,585,586]^{2}[587,} \\
600]^{2} \\
{[322 \text { to } 376]^{3},[392 \text { to } 425]^{3}} \\
{[456 \text { to } 490]^{3},[517 \text { to } 535]^{3}}\end{array}$ \\
\hline Patient 4 & $1017-65-1436$ & 594 & $\begin{array}{l}14: 36- \\
16: 15\end{array}$ & $\begin{array}{c}{[1 \text { to } 12]^{1}} \\
{[426 \text { to } 594]^{2}} \\
{[24 \text { to } 407]^{3}}\end{array}$ & $\begin{array}{c}{[24]^{1}} \\
{[21,22,23,27,28,29]^{2}} \\
{[490,497,501,507]^{2}} \\
{[510 \text { to } 513]^{2},} \\
{[178 \text { to } 227]^{3},[240 \text { to } 272]^{3}} \\
{[300 \text { to } 346]^{3},[356 \text { to } 376]^{3}} \\
{[392 \text { to } 407]^{3}}\end{array}$ \\
\hline Patient 5 & 1029-65-1010 & 576 & $\begin{array}{l}10: 09- \\
11: 45\end{array}$ & $\begin{array}{l}{[1 \text { to } 115]^{1}} \\
{[120 \text { to } 132]^{2}} \\
{[520 \text { to } 570]^{2}} \\
{[137 \text { to } 509]^{3}}\end{array}$ & $\begin{array}{c}{[29,77,116,121]^{1}} \\
{\left[[142]^{2}\right.} \\
{[285 \text { to } 303]^{3},[311 \text { to } 342]^{3}} \\
{[368 \text { to } 381]^{3},[430 \text { to } 482]^{3}}\end{array}$ \\
\hline Patient 6 & $1107-17-1334$ & 486 & $\begin{array}{c}13: 34- \\
14: 55\end{array}$ & $\begin{array}{c}{[1 \text { to } 65]^{1}} \\
{[72 \text { to } 77]^{2}} \\
{\left[[85 \text { to end }]^{3}\right.}\end{array}$ & $\begin{array}{c}{[33,49]^{1},[51 \text { to } 54]^{1}} \\
\left.[145 \text { to } 219]^{3}, 267 \text { to } 283\right]^{3} \\
{[310 \text { to } 349]^{3}}\end{array}$ \\
\hline
\end{tabular}

As shown in Table 5, NW-1 showed weak recognition (26\%) for the Class-2 'Light Anaesthesia', but showed much better accuracy for Classes 1 and 3, 'Awake' and 'Moderate Anaesthesia', respectively. The reason comes from the close values for the 3 scales of the SampEn measures. The difference between the 'Awake' and the 'Moderate Anaesthesia' is wide. Therefore, the accuracy for Class-2 was $18 \%$ and that for the remaining two classes, 'Awake' and 'Moderate anaesthesia', were $86 \%$ and $72 \%$, respectively. 
For the other two networks under study, NW-2 and NW-3, which were trained for only two levels without EMD filtering, i.e., the 'Awake' and the 'Moderate anaesthesia', the results showed more accuracy. In particular, accuracy for Class-1 and Class-3 was $74 \%$ and 91\%, respectively. For NW-3, which was trained for 2 levels with EMD filtering, the accuracy of classification reached $92 \%$ to $95 \%$.

The overall accuracy is valued as $72 \%, 90 \%$, and $95 \%$ for NW-1, NW-2, and NW-3, respectively.

\section{CONClusion}

In summary, we propose a new method to assess the DoA. The main feature of this method is the use of EMD to filter the EEG set in its generic form along with the use of MSE with SampEn and three time scales to obtain additional dimensions for better recognition of the three anaesthetic levels. Statistical analysis showed a distinct correlation between samples in the same class and distinction between the three different levels of consciousness. ANN was the sole classifier used in this method and its effectiveness was evaluated by testing on the EEG data of 14of the 20 patients. An accuracy rate of over 95\% was obtained using this method of using MSSE and EMD filtering with ANN classifier in detecting the 3 levels of DoA.

\section{REFERENCES}

[1] C. D. Kent and K. B. Domino, "Depth of anaesthesia," Current Opinion in Anaesthesiology 22.6 (2009) $782-787$.

[2] Shieh, Jiann Shing, D.a. Linkens, and J.e. Peacock. "Hierarchical rule-based and self-organizing fuzzy logic control for depth of anaesthesia." IEEE Transactions on Systems, Man and Cybernetics, Part C (Applications and Reviews) 29.1 (1999): 98-109.

[3] M. Jeanne, R. Logier, J. De Jonckheere, and B. Tavernier, "Heart rate variability during total intravenous anesthesia: effects of nociception and analgesia," Autonomic Neuroscience: Basic and Clinical, vol. 147, no. 1-2, pp. 91-96, 2009.

[4] Sigl, Jeffrey C., and Nassib G. Chamoun. "An introduction to bispectral analysis for the electroencephalogram." Journal of Clinical Monitoring 10.6 (1994): 392-404.

[5] C. Rosow and P. J. Manberg, "Bispectral index monitoring," Anesthesiology Clinics of North America, 19.4(2001) 947-966.

[6] Punjasawadwong, Y., A. Phongchiewboon, N. Bunchungmonkol, and Dn Braaksma. "Bispectral index for improving anaesthetic delivery and postoperative recovery." The Cochrane Database of Systematic Reviews (2002).

[7] Su, Cui, Zhenhu Liang, Xiaoli Li, Duan Li, Yongwang Li, and Mauro Ursino. "A Comparison of Multiscale Permutation Entropy Measures in On-Line Depth of Anesthesia Monitoring." Plos One 11.10 (2016).

[8] Benzy, V. K., and E. A. Jasmin. "A Combined Wavelet and Neural Network Based Model for Classifying Depth of Anaesthesia." Procedia Computer Science 46 (2015): 1610-1617.

[9] Lalitha, V., and C. Eswaran. "Automated detection of anesthetic depth levels using chaotic features with artificial neural networks." Journal of Medical Systems 31.6 (2007): 445-452.

[10] Srinivasan, V., C. Eswaran, and N. Sriraam. "EEG based automated detection of anesthetic levels using a recurrent artificial neural network." International Journal of Bioelectromagnetism 7.2 (2005): 267-270.

[11] Coşkun, Mustafa, et al. "Determining the appropriate amount of anesthetic gas using DWT and EMD combined with neural network." Journal of Medical Systems 39.1 (2015): 1-10.

[12] Kreuzer, Matthias, Eberhard F. Kochs, Gerhard Schneider, and Denis Jordan. "Non-stationarity of EEG during wakefulness and anaesthesia: advantages of EEG permutation entropy monitoring." Journal of Clinical Monitoring and Computing 28.6 (2014): 573-80.

[13] Costa, Madalena, Ary L. Goldberger, and C-K. Peng. "Multiscale entropy analysis of complex physiologic time series." Physical Review Letters 89.6 (2002): 068102.

[14] Ortolani, O. "EEG signal processing in anaesthesia. Use of a neural network technique for monitoring depth of anaesthesia." British Journal of Anaesthesia 88.5 (2002): 644-648.Web.

[15] Lavrov, N. G., V. V. Bulaev, E. N. Solouhin, S. A. Taratuhin, and A. V. Chistyakov. "Bispectral Analysis of Electroencephalogram Using Neural Networks to Assess the Depth of Anesthesia." Biomedical Engineering 49.6 (2016): 380-84. 
[16] Li, Tianning, and Peng Wen. "Depth of anaesthesia assessment based on adult electroencephalograph beta frequency band." Australasian Physical \& Engineering Sciences in Medicine 39.3 (2016): 773-781.

[17] Güntürkün, Rüştü. "Determining the amount of anesthetic medicine to be applied by using Elman's recurrent neural networks via resilient back propagation." Journal of Medical Systems 34.4 (2010): 493-497.

[18] Srinivasan, V., C. Eswaran, and N. Sriraam. "EEG based automated detection of anesthetic levels using a recurrent artificial neural network." International Journal of Bioelectromagnetism 7.2 (2005): 267-270.

[19] Liang, Zhenhu, Yinghua Wang, Xue Sun, Duan Li, Logan J. Voss, Jamie W. Sleigh, Satoshi Hagihira, and Xiaoli Li. "EEG entropy measures in anesthesia." Frontiers in Computational Neuroscience 9 (2015).

[20] Ghanatbari, M., A. R. Mehri Dehnavi, H. Rabbani, and A. R. Mahoori. "Estimating the depth of anesthesia by applying sub parameters to an artificial neural network during general anesthesia." 2009 9th International Conference on Information Technology and Applications in Biomedicine (2009).

[21] Musizza, Bojan, and Samo Ribaric. "Monitoring the depth of anaesthesia." Sensors 10.12 (2010): 10896-10935.

[22] Whyte, Simon David, and Peter Driscoll Booker. "Monitoring depth of anaesthesia by EEG." BJA CEPD Reviews 3.4 (2003): 106-110.

[23] Costa, Madalena, Ary L. Goldberger, and C-K. Peng. "Multiscale entropy analysis of biological signals." Physical Review E 71.2 (2005): 021906.

[24] Kreuzer, Matthias, Eberhard F. Kochs, Gerhard Schneider, and Denis Jordan. "Non-stationarity of EEG during wakefulness and anaesthesia: advantages of EEG permutation entropy monitoring." Journal of Clinical Monitoring and Computing 28.6 (2014): 573-80.

[25] Palendeng, Mario Elvis, Peng Wen, and Yan Li. "Real-time depth of anaesthesia assessment using strong analytical signal transform technique." Australasian Physical \& Engineering Sciences in Medicine 37.4 (2014): 723-730.

[26] Manilo, L. A., and S. S. Volkova. "Recognition of the deep anesthesia stage from parameters of the approximated entropy of EEG signal." Pattern Recognition and Image Analysis 23.1 (2013): 92-97.

[27] Schmidt, Gunter N., Petra Bischoff, Thomas Standl, Angelika Hellstern, Olaf Teuber, and Jochen Schulte Esch. "Comparative Evaluation of the Datex-Ohmeda S/5 Entropy Module and the Bispectral Index?? Monitor during Propofol???Remifentanil Anesthesia." Anesthesiology (2004): 1283-290.

[28] Viertio-Oja, H., V. Maja, M. Sarkela, P. Talja, N. Tenkanen, H. Tolvanen-Laakso, M. Paloheimo, A. Vakkuri, A. Yli-Hankala, and P. Merilainen. "Description of the Entropytm algorithm as applied in the Datex-Ohmeda S/5tm Entropy Module." Acta Anaesthesiologica Scandinavica 48.2 (2004): 154-61.

[29] Ferenets, Rain, Ann Vanluchene, Tarmo Lipping, Bj??rn Heyse, and Michel M. R. F. Struys. "Behavior of Entropy/Complexity Measures of the Electroencephalogram during Propofolinduced Sedation." Anesthesiology 106.4 (2007): 696-706.

[30] Sleigh, J. W., and J. Donovan. "Comparison of bispectral index, 95\% spectral edge frequency and approximate entropy of the EEG, with changes in heart rate variability during induction of general anaesthesia." British Journal of Anaesthesia 82.5 (1999): 666-71.

[31] Richman, J. S., and J. R. Moorman. "Physiological time-series analysis using approximate and sample entropy." American Journal of Physiology_Heart and Circulatory Physiology 278.6 (2000): H2039-2049.

[32] Bruhn, Jörgen, Heiko Röpcke, and Andreas Hoeft. "Approximate Entropy as an Electroencephalographic Measure of Anesthetic Drug Effect during Desflurane Anesthesia." Anesthesiology 92.3 (2000): 715-26.

[33] Takahashi, Tetsuya, Raymond Y. Cho, Tomoyuki Mizuno, Mitsuru Kikuchi, Tetsuhito Murata, Koichi Takahashi, and Yuji Wada. "Antipsychotics reverse abnormal EEG complexity in drugnaive schizophrenia: A multiscale entropy analysis." NeuroImage 51.1 (2010): 173-82. 
[34] G. Ouyang, C. Dang, and X. Li, "Multiscale entropy analysisof EEG recordings in epileptic rats," Biomedical Engineering:Applications, Basis and Communications, vol. 21, no. 3, pp. 169176, 2009.

[35] Liu, Quan, Qin Wei, Shou-Zen Fan, Cheng-Wei Lu, Tzu-Yu Lin, Maysam F. Abbod, and JiannShing Shieh. "Adaptive Computation of Multiscale Entropy and Its Application in EEG Signals for Monitoring Depth of Anesthesia During Surgery." Entropy 14.12 (2012): 978-92.

[36] R. Nayak, L. Jain, and B. Ting, "Artificial neural networks in biomedical engineering: a review," in Proceedings of the Asia-Pacific Conference on Advance Computation, 2001.

[37] Bruhn, Jörgen, Thomas W. Bouillon, Lucian Radulescu, Andreas Hoeft, Edward Bertaccini, and Steven L. Shafer. "Correlation of Approximate Entropy, Bispectral Index, and Spectral Edge Frequency 95 (SEF95) with Clinical Signs of "Anesthetic Depth" during Coadministration of Propofol and Remifentanil." Anesthesiology 98.3 (2003): 621-27.

[38] Wei, Qin, Quan Liu, Shou-Zhen Fan, Cheng-Wei Lu, Tzu-Yu Lin, Maysam Abbod, and JiannShing Shieh. "Analysis of EEG via Multivariate Empirical Mode Decomposition for Depth of Anesthesia Based on Sample Entropy." Entropy 15.9 (2013): 3458-470.

[39] Huang, Jeng-Rung, Shou-Zen Fan, Maysam Abbod, Kuo-Kuang Jen, Jeng-Fu Wu, and JiannShing Shieh. "Application of Multivariate Empirical Mode Decomposition and Sample Entropy in EEG Signals via Artificial Neural Networks for Interpreting Depth of Anesthesia." Entropy 15.9 (2013): 3325-339.

[40] Wei, Qin, Yang Li, Shou-Zen Fan, Quan Liu, Maysam F. Abbod, Cheng-Wei Lu, Tzu-Yu Lin, Kuo-Kuang Jen, Shang-Ju Wu, and Jiann-Shing Shieh. "A critical care monitoring system for depth of anaesthesia analysis based on entropy analysis and physiological information database." Australasian Physical \& Engineering Sciences in Medicine 37.3 (2014): 591-605.

[41] Liu, Quan, Qin Wei, Shou-Zen Fan, Cheng-Wei Lu, Tzu-Yu Lin, Maysam F. Abbod, and JiannShing Shieh. "Adaptive Computation of Multiscale Entropy and Its Application in EEG Signals for Monitoring Depth of Anesthesia During Surgery." Entropy 14.12 (2012): 978-92.

[42] Shalbaf, R., H. Behnam, J. Sleigh, and L. Voss. "Measuring the effects of sevoflurane on electroencephalogram using sample entropy." Acta Anaesthesiologica Scandinavica 56.7 (2012): 880-89.

[43] S. Samara Singhe, Neural Networks for Applied Sciences and Engineering: Form Fundamental to Complex Pattern Recognition, Taylor \& Francis, Boca Raton, Fla, USA, 2007.

[44] https://en.wikipedia.org/wiki/Sample_entropy.

[45] Su C, Liang Z, Li X, Li D, Li Y, Ursino M. “A comparison of multiscale permutation entropy measures in on-line depth of anesthesia monitoring". PloS one. 2016 Oct 10;11(10):e0164104.

[46] Trnka P, Hofreiter M. "The empirical mode decomposition in real-time", InProceedings of the 18th international conference on process control, Tatranská Lomnica, Slovakia (2011), 14-17.

\section{AUTHOR's BIOGRAPHY}

Dr. Mohamad Almeer, is a Qatari scholar. He received his $\mathrm{PhD}$ degree from the University of Wales, UK in 2000 and his Master degree from Wayne State University, Michigan, U.S.A 1996. He joined Qatar University in 2000 and chaired several committees at Qatar University and outside. He is consultant for several entities in Doha, Qatar. Dr. Almeer worked on digital systems, map and data reduction and management and published several journals papers in this area. Dr. Almeer's research interest includes, neural networks, Digital Systems, security, high performance computing. 